OCHA-SP-01-07

\title{
Construction of some missing eigenvectors of the XYZ spin chain at the discrete coupling constants and the exponentially large spectral degeneracy of the transfer matrix
}

\author{
Tetsuo Deguchi \\ Department of Physics, Faculty of Science \\ Ochanomizu University \\ 2-1-1 Ohtsuka, Bunkyo-Ku, Tokyo 112-8610, Japan .
}

\begin{abstract}
We discuss an algebraic method for constructing eigenvectors of the transfer matrix of the eight-vertex model at the discrete coupling parameters. We consider the algebraic Bethe ansatz of the elliptic quantum group $E_{\tau, \eta}\left(s l_{2}\right)$ for the case where the parameter $\eta$ satisfies $2 N \eta=m_{1}+m_{2} \tau$ for arbitrary integers $N, m_{1}$ and $m_{2}$. When $m_{1}$ or $m_{2}$ is odd, the eigenvectors thus obtained have not been discussed previously. Furthermore, we construct a family of degenerate eigenvectors of the XYZ spin chain, some of which are shown to be related to the $s l_{2}$ loop algebra symmetry of the XXZ spin chain. We show that the dimension of some degenerate eigenspace of the XYZ spin chain on $L$ sites is given by $N 2^{L / N}$, if $L / N$ is an even integer. The construction of eigenvectors of the transfer matrices of some related IRF (interaction-rounda-face) models is also discussed.
\end{abstract}

*deguchi@phys.ocha.ac.jp . 


\section{INTRODUCTION}

The exact solution [1] 엉 of the eight-vertex model has played a central role in the study of exactly solved models and integrable lattice models [0,6]. The partition function of the model was obtained by the functional method of the transfer matrix [1]. The eigenvectors of the transfer matrix of the model was constructed through the vertex-IRF correspondence [2 4 . The algebraic Bethe ansatz was formulated for the eight-vertex model [7]. The knowledge of the exact solution is also important in the continuum limits of the lattice models to the field theories and their connections to conformal field theories [8 11].

Recently, another algebraic Bethe ansatz method has been introduced for the eight-vertex model [12 [14]. The method is based on the elliptic quantum group $E_{\tau, \eta}\left(s l_{2}\right)$ [12,13], which is associated with the $R$-matrix of the eight-vertex solid-on-solid model (8VSOS model) [3,15], and is also related to Drinfeld's quasi-Hopf algebra [16.17]. In Ref. [14], however, the eigenvectors of the transfer matrix of the eight-vertex model is discussed only for the case where the parameter $\eta$ is generic. Here we note that both for the generic and discrete $\eta$ cases some eigenvectors are discussed in Refs. [2] [6], [7]. Thus, the primary purpose of this paper is to discuss the algebraic Bethe ansatz of $E_{\tau, \eta}\left(s l_{2}\right)$ at the discrete coupling constants. Then, we show that it also gives such eigenvectors of the eight-vertex model that are not discussed in Refs. [2 - 4 . 7 .

Let us discuss the coupling parameters $\tau$ and $\eta$ of $E_{\tau, \eta}\left(s l_{2}\right)$, explicitly. For an illustration, we consider the Hamiltonian of the XYZ spin chain under the periodic boundary conditions, which is given by the derivative of the homogeneous transfer matrix of the eight-vertex model

$$
H_{X Y Z}=\sum_{j=1}^{L}\left(J_{X} \sigma_{j}^{X} \sigma_{j+1}^{X}+J_{Y} \sigma_{j}^{Y} \sigma_{j+1}^{Y}+J_{Z} \sigma_{j}^{Z} \sigma_{j+1}^{Z}\right)
$$

In terms of the elliptic modulus $k$ (or $\tau$ ) and the coupling parameter $\eta$, the coupling constants of the XYZ chain are expressed as follows

$$
J_{X}=J\left(1+k \operatorname{sn}^{2}(2 \eta, k)\right), \quad J_{Y}=J\left(1-k \operatorname{sn}^{2}(2 \eta, k)\right), \quad J_{Z}=J \operatorname{cn}(2 \eta, k) \operatorname{dn}(2 \eta, k)
$$

Here $\operatorname{sn}(z, k), \operatorname{cn}(z, k)$ and $\operatorname{dn}(z, k)$ denote the Jacobian elliptic functions. For the discrete cases of Refs. [2] [, [], the eigenvectors of the transfer matrix of the eight-vertex model are constructed under the condition: $2 N \eta=4 m_{1} K+2 i m_{2} K^{\prime}$ where $N, m_{1}$ and $m_{2}$ are arbitrary integers. Here, the symbols $K$ and $K^{\prime}$ have denoted the complete elliptic integrals of the first and second kind, respectively. It has not been discussed explicitly how one can construct eigenvectors for the case of $2 N \eta=2 m_{1} K+i m_{2} K^{\prime}$ with given integers $N, m_{1}$ and $m_{2}$. Here we note that the functional relation [1] is derived under the latter condition: $2 N \eta=2 m_{1} K+i m_{2} K^{\prime}$.

There is another motivation of the paper. Recently, it has been found that the XXZ spin chain at the roots of unity has the spectral symmetry of the $s l_{2}$ loop algebra [18]. The explicit expressions of the generators commuting with the XXZ Hamiltonian under the periodic boundary conditions are given in Appendix A. Several non-trivial properties of the spectral degeneracy of the $s l_{2}$ loop algebra have been discussed [19]. Here, the Hamiltonian

of the XXZ spin chain gives the special case of the XYZ Hamiltonian with $J_{X}=J_{Y}$, which 
is obtained by taking the trigonometric limit: $k \rightarrow 0$ for the coupling constants of eq. (1.2). Furthermore, it has been suggested in Ref. [18 through a numerical study that the XYZ spin chain should have a very large spectral degeneracy similar to the $s l_{2}$ loop algebra symmetry of the XXZ spin chain. In fact, the discrete condition: $2 N \eta=2 m_{1} K+i m_{2} K^{\prime}$ of the XYZ spin chain corresponds to the roots of unity condition of the XXZ Hamiltonian, in the trigonometric limit. Therefore, the construction of eigenvectors of the XYZ spin chain at the discrete coupling constants should be important in studying the conjectured spectral degeneracy of the XYZ spin chain.

The outline of the paper is given in the following. In $\S 2$ we introduce the elliptic quantum group $E_{\tau, \eta}\left(s l_{2}\right)$, briefly. In the paper we employ almost the same symbols with Ref. 114 for $E_{\tau, \eta}\left(s l_{2}\right)$. In $\S 3$ we show the main results of the algebraic Bethe ansatz of $E_{\tau, \eta}\left(s l_{2}\right)$ for the discrete $\eta$ case of $2 N \eta=m_{1}+m_{2} \tau$, which corresponds to the case of $2 N \eta=2 m_{1} K+i m_{2} K^{\prime}$ in the notation of Refs. [1]. Then, we obtain a slightly generalized expression for the eigenvalues of the transfer matrix expressed in terms of rapidities. In $\S 4$, for the discrete $\eta$ case, we discuss some important points in constructing eigenvectors for the 8VSOS model and its two variants: the ABF (or 8VRSOS) model and the cyclic SOS model [20 22], and then for the eight-vertex model. In $\S 5$, we formulate an algebraic method for constructing degenerate eigenvectors of the transfer matrix of the eight-vertex model. It indeed gives a large number of degenerate eigenvectors. For an illustration, we calculate analytically the dimension of the largest degenerate eigenspace of the XYZ model defined on the $L$ sites. We show that it is given by $N 2^{L / N}$, if $L / N$ is an even integer. Thus we see that the spectral degeneracy can increase exponentially with respect to the lattice size $L$. Finally, in $\S 6$, we give some discussions. In order to make the paper self-consistent, some appendices are provided. In Appendix A, we review the $s l_{2}$ loop algebra symmetry of the XXZ spin chain at the root of unity. In Appendix B, we explain the evaluation modules of the elliptic quantum group $E_{\tau, \eta}\left(s l_{2}\right)$. Finally in Appendix C, we give the Boltzmann weights of the RSOS models associated with the eight-vertex model.

\section{ELLIPTIC QUANTUM GROUP $E_{\tau, \eta}\left(S L_{2}\right)$}

The elliptic quantum group $E_{\tau, \eta}\left(s l_{2}\right)$ is an algebra generated by meromorphic functions of a variable $h$ and the matrix elements of an operator-valued matrix $L(z, \lambda)$ with noncommutative entries [12 14], which satisfy the Yang-Baxter relation with a dynamical shift

$$
\begin{aligned}
& R^{(12)}\left(z_{12}, \lambda-2 \eta h^{(3)}\right) L^{(1)}\left(z_{1}, \lambda\right) L^{(2)}\left(z_{2}, \lambda-2 \eta h^{(1)}\right) \\
= & L^{(2)}\left(z_{2}, \lambda\right) L^{(1)}\left(z_{1}, \lambda-2 \eta h^{(2)}\right) R^{(12)}\left(z_{12}, \lambda\right)
\end{aligned}
$$

Here $h$ is a generator of the Cartan subalgebra $\mathbf{h}$ of $s l_{2}$.

Let us formulate the $R$-matrix of the elliptic quantum group $E_{\eta, \tau}\left(s l_{2}\right)$, explicitly. We introduce the theta function

$$
\theta(z ; \tau)=2 p^{1 / 4} \sin \pi z \prod_{n=1}^{\infty}\left(1-p^{2 n}\right)\left(1-p^{2 n} \exp (2 \pi i z)\right)\left(1-p^{2 n} \exp (-2 \pi i z)\right),
$$

where the nome $p$ is related to the parameter $\tau$ by $p=\exp (\pi i \tau)$ with $\operatorname{Im} \tau>0$. Let $V$ be the two-dimensional complex vector space with the basis $e[1]$ and $e[-1]$. Here we denote 
$e[-1]$ also as $e[2]$, and let $E_{i j}$ denote the matrix satisfying $E_{i j} e[k]=\delta_{j k} e[i]$. Then, the $R$-matrix $R(z, \lambda) \in \operatorname{End}(V)$ is given by

$$
\begin{aligned}
R(z, \lambda ; \eta, \tau)= & E_{11} \otimes E_{11}+E_{22} \otimes E_{22}+\alpha(z, \lambda) E_{11} \otimes E_{22} \\
& +\beta(z, \lambda) E_{12} \otimes E_{21}+\beta(z,-\lambda) E_{21} \otimes E_{12}+\alpha(z,-\lambda) E_{22} \otimes E_{11}
\end{aligned}
$$

where $h=E_{11}-E_{22}$ and $\alpha(z, \lambda)$ and $\beta(z, \lambda)$ are defined by

$$
\alpha(z, \lambda)=\frac{\theta(z) \theta(\lambda+2 \eta)}{\theta(z-2 \eta) \theta(\lambda)}, \quad \beta(z, \lambda)=-\frac{\theta(z+\lambda) \theta(2 \eta)}{\theta(z-2 \eta) \theta(\lambda)} .
$$

The elliptic quantum group $E_{\tau, \eta}\left(s l_{2}\right)$ has two types of generators: (i) meromorphic functions of $f(h)$ of one-variable with period $1 / \eta$; (ii) the non-commutative matrix elements of the $L$ operator: $a(z, \lambda), b(z, \lambda), c(z, \lambda)$, and $d(z, \lambda)$, which satisfy the relations arising from (2.1), such as shown in the following

$$
\begin{aligned}
& b\left(z_{1}, \lambda\right) b\left(z_{2}, \lambda+2 \eta\right)=b\left(z_{2}, \lambda\right) b\left(z_{1}, \lambda+2 \eta\right) \\
& b\left(z_{1}, \lambda\right) a\left(z_{2}, \lambda+2 \eta\right)=b\left(z_{2}, \lambda\right) a\left(z_{1}, \lambda+2 \eta\right) \beta\left(z_{1}-z_{2}, \lambda\right)+a\left(z_{2}, \lambda\right) b\left(z_{1}, \lambda-2 \eta\right) \alpha\left(z_{1}-z_{2},-\lambda\right)
\end{aligned}
$$

Hereafter, we suppress the $\lambda$-dependence of the operators $a(z, \lambda), b(z, \lambda), c(z, \lambda)$ and $d(z, \lambda)$, for simplicity.

The definition of the evaluation Verma module for the elliptic quantum group $E_{\tau, \eta}\left(s l_{2}\right)$ is explicitly given in Appendix B.

\section{ALGEBRAIC BETHE ANSATZ AT THE DISCRETE COUPLING PARAMETERS}

Let $W=V_{\Lambda_{1}}\left(z_{1}\right) \otimes \cdots \otimes V_{\Lambda_{n}}\left(z_{n}\right)$ be the tensor product of evaluation modules $V_{\Lambda_{j}}\left(z_{j}\right)$ 's [14] (See also Appendix B). The total spin $\Lambda$ is given by $\Lambda=\Lambda_{1}+\Lambda_{2}+\cdots+\Lambda_{n}$. Here, we assume $\Lambda$ is given by $\Lambda=2 \ell$ or $\Lambda=2 \ell+1$ with an integer $\ell$. The transfer matrix $T(z)$ of the elliptic algebra is given by the trace of the $L$-operator acting on the tensor product space $W$. We denote by $v_{0}$ the highest weight vector of $W: h v_{0}=\Lambda v_{0}$.

Let us assume hereafter that the coupling parameter $\eta$ is given by the discrete values: $2 N \eta=m_{1}+m_{2} \tau$ for some integers $N, m_{1}$ and $m_{2}$. We now take an integer $m$ satisfying the following condition

$$
2 m=\Lambda-r N, \quad \text { for } \quad r \in \mathbf{Z} .
$$

We consider the $m$ th product of the creation operators: $b\left(t_{1}\right) \cdots b\left(t_{m}\right)$ with $m$ rapidities $t_{1}, \ldots, t_{m}$. Here we note that the integers $m$ and $\ell$ can be different, while in Ref. [14] only the case of $m=\ell$ and generic $\eta$ is discussed. Let us define a vector $v_{c}$ by $v_{c}=g_{c}(\lambda) v_{0}$ where $g_{c}(\lambda)$ is given by

$$
g_{c}(\lambda)=e^{c \lambda} \prod_{j=1}^{m} \frac{\theta(\lambda-2 \eta j)}{\theta(2 \eta)}
$$


Let us first consider the case when $m_{2}=0$. Then, through the fundamental commutation relations such as shown in eq. (2.5), we can show

$T(w) b\left(t_{1}\right) \cdots b\left(t_{m}\right) v_{c}=C_{0}(w) b\left(t_{1}\right) \cdots b\left(t_{m}\right) v_{c}+\sum_{j=1}^{m} C_{j} b\left(t_{1}\right) \cdots b\left(t_{j-1}\right) b(w) b\left(t_{j+1}\right) \cdots b\left(t_{m}\right) v_{c}$.

Here the coefficients $C_{0}$ and $C_{j}$ are given by

$$
\begin{aligned}
C_{0}(w) & =e^{-2 \eta c} \prod_{j=1}^{m} \frac{\theta\left(w-t_{j}+2 \eta\right)}{\theta\left(w-t_{j}\right)}+(-1)^{r m_{1}} e^{2 \eta c} \prod_{j=1}^{m} \frac{\theta\left(w-t_{j}-2 \eta\right)}{\theta\left(w-t_{j}\right)} \prod_{\alpha=1}^{n} \frac{\theta\left(w-p_{\alpha}\right)}{\theta\left(w-q_{\alpha}\right)} \\
C_{j} & =e^{-2 \eta c} \frac{\theta(2 \eta)}{\theta\left(t_{j}-w\right)} \frac{\theta\left(t_{j}-w+\lambda\right)}{\theta(\lambda)}\left(\prod_{k=1 ; k \neq j}^{m} f_{k j}-(-1)^{r m_{1}} e^{4 \eta c} \prod_{k=1 ; k \neq j}^{m} f_{j k} \prod_{\alpha=1}^{n} \frac{\theta\left(t_{j}-p_{\alpha}\right)}{\theta\left(t_{j}-q_{\alpha}\right)}\right)
\end{aligned}
$$

where $p_{\alpha}=z_{\alpha}+\eta\left(-\Lambda_{\alpha}+1\right)$ and $q_{\alpha}=z_{\alpha}+\eta\left(\Lambda_{\alpha}+1\right)$ for $\alpha=1, \ldots n$, and the symbol $f_{j k}$ denotes the following

$$
f_{j k}=\frac{\theta\left(t_{j}-t_{k}-2 \eta\right)}{\theta\left(t_{j}-t_{k}\right)}
$$

Thus, we have found that $b\left(t_{1}\right) b\left(t_{2}\right) \cdots b\left(t_{m}\right) v$ is an eigenvector of the transfer matrix $T(z)$ with the eigenvalue $C_{0}(z)$, if rapidities $t_{1}, t_{2}, \ldots, t_{m}$ satisfy the Bethe ansatz equations

$$
\prod_{k=1}^{n} \frac{\theta\left(t_{j}-p_{k}\right)}{\theta\left(t_{j}-q_{k}\right)}=(-1)^{r m_{1}} e^{-4 \eta c} \prod_{k=1 ; k \neq j}^{m} \frac{\theta\left(t_{j}-t_{k}+2 \eta\right)}{\theta\left(t_{j}-t_{k}-2 \eta\right)} \quad \text { for } \quad j=1, \ldots, m
$$

We remark that the factor $(-1)^{r m_{1}}$ can be important for the case when $m_{1}$ is odd, which has not been discussed in the references [2, 4, 7]

When $m_{2} \neq 0$, we consider the "renormalization" of the theta function [2]

$$
\tilde{\theta}(z)=\theta(z) \exp \left(\pi m_{2}(z-1 / 2)^{2} /(2 N \eta)\right)
$$

Then we have

$$
\tilde{\theta}(z+2 N \eta)=(-1)^{m_{1}\left(m_{2}+1\right)} \tilde{\theta}(z)
$$

Replacing all the theta functions $\theta(z)$ 's in the above discussions with the renormalized ones $\tilde{\theta}(z)$ 's, for the case of $2 N \eta=m_{1}+m_{2} \tau$, we have

$$
\begin{aligned}
T(w) b\left(t_{1}\right) \cdots b\left(t_{m}\right) v_{c}= & \tilde{C}_{0}(w) b\left(t_{1}\right) \cdots b\left(t_{m}\right) v_{c} \\
& +\sum_{j=1}^{m} \tilde{C}_{j} b\left(t_{1}\right) \cdots b\left(t_{j-1}\right) b(w) b\left(t_{j+1}\right) \cdots b\left(t_{m}\right) v_{c} .
\end{aligned}
$$

Here the coefficients $\tilde{C}_{0}$ and $\tilde{C}_{j}$ are given by the following 


$$
\begin{aligned}
\tilde{C}_{0}(w)= & e^{-2 \eta c} \prod_{j=1}^{m} \frac{\tilde{\theta}\left(w-t_{j}+2 \eta\right)}{\tilde{\theta}\left(w-t_{j}\right)}+(-1)^{r m_{1}\left(m_{2}+1\right)} e^{2 \eta c} \prod_{j=1}^{m} \frac{\tilde{\theta}\left(w-t_{j}-2 \eta\right)}{\tilde{\theta}\left(w-t_{j}\right)} \prod_{\alpha=1}^{n} \frac{\tilde{\theta}\left(w-p_{\alpha}\right)}{\tilde{\theta}\left(w-q_{\alpha}\right)} \\
\tilde{C}_{j}= & e^{-2 \eta c} \frac{\tilde{\theta}(2 \eta)}{\tilde{\theta}\left(t_{j}-w\right)} \frac{\tilde{\theta}\left(t_{j}-w+\lambda\right)}{\tilde{\theta}(\lambda)} \\
& \times\left(\prod_{k=1 ; k \neq j}^{m} \tilde{f}_{k j}-(-1)^{r m_{1}\left(m_{2}+1\right)} e^{4 \eta c} \prod_{k=1 ; k \neq j}^{m} \tilde{f}_{j k} \prod_{\alpha=1}^{n} \frac{\tilde{\theta}\left(t_{j}-p_{\alpha}\right)}{\tilde{\theta}\left(t_{j}-q_{\alpha}\right)}\right)
\end{aligned}
$$

where $\tilde{f}_{j k}=\tilde{\theta}\left(t_{j}-t_{k}-2 \eta\right) / \tilde{\theta}\left(t_{j}-t_{k}\right)$. Thus, we have shown that $b\left(t_{1}\right) \cdots b\left(t_{m}\right) v$ is an eigenvector of the transfer matrix $T(z)$ with the eigenvalue $\tilde{C}_{0}(z)$, if rapidities $t_{1}, \ldots, t_{m}$ satisfy the Bethe ansatz equations

$$
\prod_{k=1}^{n} \frac{\tilde{\theta}\left(t_{j}-p_{k}\right)}{\tilde{\theta}\left(t_{j}-q_{k}\right)}=(-1)^{r m_{1}\left(m_{2}+1\right)} e^{-4 \eta c} \prod_{k=1 ; k \neq j}^{m} \frac{\tilde{\theta}\left(t_{j}-t_{k}+2 \eta\right)}{\tilde{\theta}\left(t_{j}-t_{k}-2 \eta\right)} \quad \text { for } \quad j=1, \ldots, m .
$$

We remark that the factor $(-1)^{r m_{1}\left(m_{2}+1\right)}$ should be important when $m_{1}$ is odd and $m_{2}$ is even.

Hereafter in the paper, we discuss only the case of $m_{2}=0\left(2 N \eta=m_{1}\right)$ to each of the topics, for simplicity. However, the case of $m_{2} \neq 0$ can be discussed similarly by using the renormalized theta function (3.7).

\section{THE EIGHT-VERTEX MODEL AND RELATED IRF MODELS}

\section{A. The 8VSOS and 8VRSOS models}

Let us discuss for the discrete $\eta$ case of $E_{\tau, \eta}\left(s l_{2}\right)$ how one can construct eigenvectors of the transfer matrices of the 8VSOS model [3] and its restricted versions, the ABF (or 8VRSOS) model [15] and the cyclic SOS model (8VCSOS model) [20 22], briefly. The RSOS models associated with the eight-vertex model are reviewed in Appendix C.

Let us assume that $\Lambda_{1}=\cdots=\Lambda_{n}=1$, hereafter in the paper. Thus, we have $n=\Lambda$ which is given by $2 \ell$ or $2 \ell+1$ for an integer $\ell$. We also have $n=L$, where $L$ is the lattice size defined in $\S 1$. We shall denote $n$ and $\Lambda$ by $L$, hereafter.

We introduce the $L$-operator [14] for the tensor product $W=V^{\otimes L}$

$$
L(z, \lambda)=R^{(01)}\left(z-z_{1}, \lambda-2 \eta \sum_{j=2}^{L} h^{(j)}\right) R^{(02)}\left(z-z_{2}, \lambda-2 \eta \sum_{j=3}^{L} h^{(j)}\right) \cdots R^{(0 L)}\left(z-z_{L}, \lambda\right)
$$

Here we recall that the transfer matrix $T(z)$ of $E_{\tau, \eta}\left(s l_{2}\right)$ for $W$ is given by the trace of the $L$-operator: $T(z, \lambda)=\operatorname{tr}_{0} L(z, \lambda)$.

The $R$-matrix $R(z, \lambda)$ of $E_{\eta, \tau}\left(s l_{2}\right)$ (which has been given in eq. (2.3) ) is related to the Boltzmann weights $w(a, b, c, d ; z)$ of the 8VSOS model [3] through the following relation [14]

$$
R(z,-2 \eta d) e[c-d] \otimes e[b-c]=\sum_{a} w(a, b, c, d ; z) e[b-a] \otimes e[a-d]
$$


Here $a, b, c, d$ denote the spin variables of the IRF (the Interaction Round a Face) model, which take integer values [3]. The spin variables have the constraint that the difference between the values of two nearest-neighboring spins should be given by \pm 1 . Through the relation (4.2), we can show that the transfer matrix $T(z)$ of $E_{\tau, \eta}\left(s l_{2}\right)$ acting on the "path basis" corresponds to that of the 8VSOS model [14]. Here we note that a "path" is given by a sequence of the values of spin variables satisfying the constraints on adjacent spins. Thus, if we express the eigenvector $b\left(t_{1}\right) \cdots b\left(t_{m}\right) v_{c}$ of $E_{\tau, \eta}\left(s l_{2}\right)$ in terms of the path basis [3, 14], then it gives that of the transfer matrix of the 8VSOS model.

For the 8 VRSOS model, the values of the spin variables are restricted into $N$ values such as $0,1, \ldots$, and $N-1$ [15]. In the model, it is not allowed for any pair of neighboring spins to have the values 0 and $N-1$, respectively. Thus, only such paths of the 8VSOS model that satisfy the conditions are allowed as paths of the 8VRSOS model. The transfer matrix of the $8 \mathrm{VRSOS}$ model is defined on the restricted space of paths. We can construct eigenvectors of 8VRSOS model in the restricted space of paths.

For the 8VSOS and ABF models, the periodic boundary conditions on the path basis are satisfied only when $L=2 \mathrm{~m}$. Thus, we can construct the eigenvectors of the models only for the case when $L$ is even.

For the cyclic SOS (or 8VCSOS) model [20 22], the spin variables take the restricted values such as $0,1, \ldots, N-1$, where the values 0 and $N-1$ are allowed for any pair of neighboring spins. Thus, the admissibility condition on the spin values can be expressed by a cyclic graph of $N$ nodes. The periodic boundary conditions on the path basis of the cyclic SOS model are satisfied under the constraint: $L-2 m=r N$, where $r$ can be non-zero integers. Therefore, for the cyclic SOS model, we can consider eigenvectors also for the odd lattice-size case. For instance, we may take $L=7$ and consider the case of $N=3, r=1$ and $m=2$, which satisfies the constraint: $L-2 m=r N$. Furthermore, when $2 N \eta=m_{1}$ with $m_{1}$ odd, then the factor $(-1)^{r m_{1}}$ in the formula of eigenvalues (3.4) is given by -1 , not by 1 .

\section{B. The eight-vertex model}

Let us introduce the theta functions $\theta_{0}(z)$ and $\theta_{1}(z)$ satisfying $\theta_{\alpha}(z+1)=(-1)^{\alpha} \theta_{\alpha}(z)$ and $\theta_{\alpha}(z+\tau)=i e^{-\pi i(z+\tau / 2)} \theta_{1-\alpha}(z)$ for $\alpha=0,1$, where we define $\theta_{1}(z)$ by $\theta_{1}(z, \tau)=\theta(z, 2 \tau)$ [14]. In terms of the theta functions, the $R$-matrix of the eight-vertex model [四] is given by

$$
\begin{aligned}
R_{8 V}(z)= & a_{8 V}(z)\left(E_{11} \otimes E_{11}+E_{22} \otimes E_{22}\right)+b_{8 V}(z)\left(E_{11} \otimes E_{22}+E_{22} \otimes E_{11}\right) \\
& +c_{8 V}(z)\left(E_{12} \otimes E_{21}+E_{21} \otimes E_{12}\right)+d_{8 V}(z)\left(E_{12} \otimes E_{12}+E_{21} \otimes E_{21}\right)
\end{aligned}
$$

where the Boltzmann weights $a_{8 V}(z), b_{8 V}(z), c_{8 V}(z)$ and $d_{8 V}(z)$ are expressed as

$$
\begin{aligned}
& a_{8 V}(z)=\frac{\theta_{0}(z) \theta_{0}(2 \eta)}{\theta_{0}(z-2 \eta) \theta_{0}(0)}, \quad b_{8 V}(z)=\frac{\theta_{1}(z) \theta_{0}(2 \eta)}{\theta_{1}(z-2 \eta) \theta_{0}(0)}, \\
& c_{8 V}(z)=-\frac{\theta_{0}(z) \theta_{1}(2 \eta)}{\theta_{1}(z-2 \eta) \theta_{0}(0)}, \quad d_{8 V}(z)=-\frac{\theta_{1}(z) \theta_{1}(2 \eta)}{\theta_{0}(z-2 \eta) \theta_{0}(0)} .
\end{aligned}
$$

The transfer matrix $T_{8 V}(z)$ of the eight-vertex model is given by the trace over the 0th vector space: $T_{8 V}(z)=\operatorname{tr}_{0} L_{8 V}(z)$, where the operator $L_{8 V}(z)$ is defined by 


$$
L_{8 V}(z)=R_{8 V}\left(z-z_{1}\right)^{(01)} \cdots R_{8 V}\left(z-z_{L}\right)^{(0 L)}
$$

Let us consider the correspondence between the eight-vertex model and the 8VSOS model [3]. We introduce some symbols of Ref. [14] for $E_{\tau, \eta}\left(s l_{2}\right)$. We define the following matrix

$$
S(z, \lambda)=\left(\begin{array}{cc}
\theta_{0}(z-\lambda+1 / 2) & -\theta_{0}(-z-\lambda+1 / 2) \\
-\theta_{1}(z-\lambda+1 / 2) & \theta_{1}(-z-\lambda+1 / 2)
\end{array}\right)
$$

Some essential part of the vertex-IRF correspondence [3] is expressed in terms of the matrices

$$
S(w, \lambda)^{(2)} S\left(z, \lambda-2 \eta h^{(2)}\right)^{(1)} R(z-w, \lambda)=R_{8 V}(z-w) S(z, \lambda)^{(1)} S\left(w, \lambda-2 \eta h^{(1)}\right)^{(2)} .
$$

Here the symbol $S(z, \lambda)^{(j)}$ denotes the matrix $S(z, \lambda)$ acting on the $j$ th space $V\left(z_{j}\right)$ of the tensor product $W=V^{\otimes L}$. Next, we consider the tensor product of the matrices acting on $W$

$$
S_{L}(\lambda)=S\left(z_{L}, \lambda\right)^{(L)} S\left(z_{L-1}, \lambda-2 \eta h^{(L)}\right)^{(L-1)} \cdots S\left(z_{1}, \lambda-2 \eta \sum_{j=2}^{L} h^{(j)}\right)^{(1)}
$$

Then, through the vertex-IRF correspondence [3, 14], we have

$$
L_{8 V}(z) S(z, \lambda)^{(0)} S_{L}\left(\lambda-2 \eta h^{(0)}\right)=S_{L}(\lambda) S(z, \lambda-2 \eta h)^{(0)} L(z, \lambda),
$$

where $h=\sum_{j=1}^{L} h^{(j)}$. Let us denote by the symbol $u_{c}$ the eigenvector $b\left(t_{1}\right) \cdots b\left(t_{m}\right) v_{c}$ constructed in $\S 3$. Here we assume that the rapidities $t_{1}, \ldots, t_{m}$ satisfy the Bethe ansatz equations (3.6). We also note that $h u_{c}=(L-2 m) u_{c}=r N u_{c}$. When $r m_{1}$ is even, we can show

$$
T_{8 V}(z) S_{L}(\lambda) u_{c}=S_{L}(\lambda+2 \eta) a(z, \lambda+2 \eta) u_{c}+S_{L}(\lambda-2 \eta) d(z, \lambda-2 \eta) u_{c} .
$$

Let us now define the following

$$
\Phi_{c}(\lambda)=\sum_{k=0}^{2 N-1} S_{L}(\lambda+2 \eta k) u_{c}(\lambda+2 \eta k),
$$

where the parameter $c$ satisfies the condition: $\exp (4 N \eta c)=1$. Here we note that in the construction (4.11) of $\Phi_{c}$, we have assumed that $u_{c}(\lambda+4 N \eta c)=u_{c}(\lambda)$. It follows from (4.10) that $\Phi_{c}(\lambda)$ gives an eigenvector of the transfer matrix $T_{8 V}(z)$ with the eigenvalue $C_{0}(z)$ defined by eq. (3.4): $T_{8 V}(z) \Phi_{c}=C_{0}(z) \Phi_{c}$. Thus, we have discussed the construction of eigenvectors for the discrete $\eta$ case of $2 N \eta=m_{1}$, when $L-2 m=r N(r \in \mathbf{Z})$ and $r m_{1}$ is even. Here we recall $W=V^{\otimes L}$ and $\Lambda=n=L$.

Let us discuss the possible connection of the present method for constructing eigenvectors of the eight-vertex model to that of Ref. [2 [4]. In fact, it is not clear yet how Baxter's method for the the general case discussed in Refs. [3, [4 is related to the present method explicitly. We first note that the parameter $\lambda$ in the paper should correspond to the parameter $s$ or $t$ of Baxter's eigenvectors in Refs. [2 国. However, it seems that there is a large difference between the ways how to control the parameter $\lambda$ or $s$ for the two methods. Thus, although there could exist more intrinsic connections between the two methods, we can only point out here that some important relations are common to both of them as mathematical formulas, such as the vertex-IRF correspondence (4.7) and the sum over the eigenvectors in eq. (4.11). Furthermore, we can compare some examples of eigenvectors constructed by the two methods, explicitly. For instance, the eigenvector constructed in Ref. [2] corresponds to the eigenvector $\Phi_{c}$ with $m=0$ and $c=0$ for the case of $2 N \eta=2 m_{1}+2 m_{2} \tau$ in the paper. 


\section{DEGENERATE EIGENVECTORS}

Let us construct degenerate eigenvectors for the transfer matrix of the eight-vertex model. We can also construct eigenvectors of the 8VSOS, ABF and cyclic SOS models similarly by the method. First, we recall that the number $m$ of the $b$ operators in the product $b\left(t_{1}\right) \cdots b\left(t_{m}\right) v_{c}$ satisfies the condition (3.1): $L-2 m=r N$. Here we note that $r m_{1}$ is even for the eight-vertex model, and also that $r=0$ for the 8VSOS and ABF models.

Let us now assume that out of $m$ rapidities $t_{1}, \ldots, t_{m}$, the first $R$ rapidities $t_{j}$ for $j=$ $1, \ldots, R$ are of standard ones satisfying the Bethe ansatz equations (3.6) with $m$ replaced by $R$, while the remaining $N F$ rapidities are formal solutions given by

$$
t_{(\alpha, j)}=t_{(\alpha)}+\eta(2 j-N-1)+\epsilon_{\alpha} r_{j}^{(\alpha)}, \quad \text { for } \quad j=1, \ldots, N \text {. }
$$

Here $\epsilon_{\alpha}$ 's are free parameters, and we shall consider the limit of sending $\epsilon_{\alpha}$ to zero, later. We call the set of $N$ rapidities $t_{(\alpha, 1)}, \ldots, t_{(\alpha, N)}$, the complete $N$-string with center $t_{(\alpha)}$. Here the index $\alpha$ runs from 1 to $F$. Furthermore, we assume that the index $(\alpha, j)$ corresponds to the number $R+N(\alpha-1)+j$ for $1 \leq \alpha \leq F$ and $1 \leq j \leq N$. Here we note that the importance of complete $N$-strings has been recently discussed for the $s l_{2}$ loop algebra symmetry of the six-vertex model [19], while for the eight-vertex model the complete strings have been suggested in Ref. [4] briefly in another context.

When $\epsilon_{\alpha}$ 's are not zero, through the commutation relations such as shown in eq. (2.5), we can show the following relation

$$
\begin{aligned}
T(z) b\left(t_{1}\right) \cdots b\left(t_{R+N F}\right) v_{c} & =C_{0}(z) b\left(t_{1}\right) \cdots b\left(t_{R+N F}\right) v_{c} \\
& +\left(\sum_{j=1}^{R}+\sum_{j=R+1}^{R+N F}\right) C_{j} b\left(t_{1}\right) \cdots b\left(t_{j-1}\right) b(z) b\left(t_{j+1}\right) \cdots b\left(t_{R+N F}\right) v_{c} .
\end{aligned}
$$

Now, let us consider the limit of sending $\epsilon_{\alpha}$ to zero. If we naively take the zero limits for $\epsilon_{\alpha}$ 's, then the convergence of the R.H.S. of eq. (5.2) is not certain. We specify the limit as follows: setting $\epsilon_{\alpha}=\epsilon$, we divide eq. (5.2) by $\epsilon^{F}$, and send $\epsilon$ to zero. Then, we can show that each of the terms of eq. (5.2) indeed converges, by making use of the following formula

$$
\prod_{1 \leq \alpha<\beta \leq m} f_{P \alpha P \beta}=\prod_{1 \leq \alpha<\beta \leq m} f_{\alpha \beta} \times \prod_{1 \leq j<k \leq m}\left(\frac{\theta\left(t_{j}-t_{k}+2 \eta\right)}{\theta\left(t_{j}-t_{k}-2 \eta\right)}\right)^{H\left(P^{-1} j-P^{-1} k\right)}, \text { for } P \in \mathcal{S}_{m}
$$

Here $H(x)$ denotes the Heaviside step function: $H(x)=1$ for $x>0, H(x)=0$ otherwise. The symbol $P \in \mathcal{S}_{m}$ denotes an element $P$ of the symmetric group of $m$ elements, where $j$ is sent to $P j \in\{1,2, \ldots, m\}$ for $j=1, \ldots m$. We recall that $f_{j k}=\theta\left(t_{j}-t_{k}-2 \eta\right) / \theta\left(t_{j}-t_{k}\right)$. The formula (5.3) has been proven in Ref. [23].

Let us consider an explicit formula describing the "matrix elements" of $b\left(t_{1}\right) \cdots b\left(t_{m}\right) v_{c}$, which has been derived in Ref. [14] for the case of $\Lambda=2 m$ and generic $\eta$. We can show that almost the same formula is valid also for the case of $\Lambda-2 m \neq 0$ when $2 N \eta=m_{1}+m_{2} \tau$. For the tensor product $W=V^{\otimes L}$, we have 


$$
\begin{aligned}
& b\left(t_{1}\right) \cdots b\left(t_{m}\right) v_{c}=(-1)^{m} e^{c(\lambda+2 \eta m)} \sum_{P \in \mathcal{S}_{m}} \sum_{1 \leq j_{1}<\cdots<j_{m} \leq L}\left(\prod_{\alpha=1}^{m} \prod_{\beta=j_{\alpha}+1}^{L} \frac{\theta\left(t_{P \alpha}-z_{\beta}\right)}{\theta\left(t_{P \alpha}-z_{\beta}-2 \eta\right)}\right) \\
& \times \prod_{1 \leq \alpha<\beta \leq m} f_{P \alpha P \beta} \times \prod_{\alpha=1}^{m} \frac{\theta\left(\lambda+t_{P \alpha}-z_{j_{\alpha}}-2 \eta\left(L-2 m-j_{\alpha}+\alpha\right)\right)}{\theta\left(t_{P \alpha}-z_{j_{\alpha}}-2 \eta\right)} \sigma_{j_{1}}^{-} \cdots \sigma_{j_{m}}^{-}|0\rangle
\end{aligned}
$$

Here $\sigma_{j}^{-}$denotes the Pauli matrix $\sigma^{-}$acting on the $j$ th vector space, and $|0\rangle$ the vacuum vector with all spins up. Here we also note that $p_{k}=z_{k}$ and $q_{k}=z_{k}+2 \eta$ since $\Lambda_{k}=1$ for $k=1, \ldots, L$.

We now discuss the behaviors of the terms of eq. (5.2) under the limit of $\epsilon$ sent to zero. In the limit, the factors $\prod_{1 \leq \alpha<\beta \leq m} f_{P \alpha P \beta}$ play the most important role in eq. (5.4). With the formula (5.3), we can single out the vanishing factors from the expression. We can show that the second part of (5.3) converges in the limit, which is given by $\prod_{1 \leq j<k \leq m}\left(\theta\left(t_{j}-t_{k}+2 \eta\right) / \theta\left(t_{j}-t_{k}-2 \eta\right)\right)^{H\left(P^{-1} j-P^{-1} k\right)}$. For the complete strings $t_{(\alpha, j)}$ with $1 \leq j \leq N$, we have specified their ordering such that $t_{(\alpha, j)}-t_{(\alpha, k)}=2 \eta(j-k)+O(\epsilon)$ for $1 \leq j<k \leq N$. Thus, the factor $\theta\left(t_{(\alpha, j)}-t_{(\alpha, k)}+2 \eta\right) / \theta\left(t_{(\alpha, j)}-t_{(\alpha, k)}-2 \eta\right)$ for an ordered pair of $j<k$ never diverges except when $j=1$ and $k=N$. If $P^{-1}(\alpha, 1)>P^{-1}(\alpha, N)$, then we have ordered pairs of $(\alpha, j)$ and $(\alpha, j+1)$ for some $j$ 's such that $P^{-1}(\alpha, j)>P^{-1}(\alpha, j+1)$, whose factors cancel out the vanishing denominator: $\theta\left(t_{1}-t_{N}-2 \eta\right)$. Here we note that $t_{(\alpha, j)}-t_{(\alpha, j+1)}+2 \eta=O(\epsilon)$.

Let us denote by $r_{a, b}^{(\alpha)}$ the difference: $r_{a, b}^{(\alpha)}=r_{a}^{(\alpha)}-r_{b}^{(\alpha)}$. We can show that all the terms of R.H.S. of eq. (5.2) converge under the limit $\epsilon \rightarrow 0$, if $r_{a, b}^{(\alpha)}$, s satisfy the following

$$
\frac{r_{a-1, a}^{(\alpha)}}{r_{a, a+1}^{(\alpha)}}=(-1)^{r m_{1}} e^{-4 \eta c} \prod_{k=1}^{R} \frac{\theta\left(t_{(\alpha, a)}-t_{k}+2 \eta\right)}{\theta\left(t_{(\alpha, a)}-t_{k}-2 \eta\right)} \prod_{\beta=1}^{L} \frac{\theta\left(t_{(\alpha, a)}-z_{\beta}-2 \eta\right)}{\theta\left(t_{(\alpha, a)}-z_{\beta}\right)}, \quad \text { for } \quad a=1, \ldots, N .
$$

Here we have assumed for $a=N$ and $a=1$ the L.H.S. of (5.5) denotes $r_{N-1, N}^{(\alpha)} / r_{N, 1}^{(\alpha)}$ and $r_{N, 1}^{(\alpha)} / r_{1,2}^{(\alpha)}$, respectively. The center $t_{(\alpha)}$ of the complete $N$-string should satisfy the two constraints on $r_{a, b}^{(\alpha)}$, s that are expressed in terms of the center $t_{(\alpha)}$ through eq. (5.5), as follows

$$
\begin{aligned}
& \frac{r_{1,2}^{(\alpha)}}{r_{N, 1}^{(\alpha)}}+\frac{r_{2,3}^{(\alpha)}}{r_{N, 1}^{(\alpha)}}+\cdots+\frac{r_{N-1, N}^{(\alpha)}}{r_{N, 1}^{(\alpha)}}+1=0, \\
& \frac{r_{1,2}^{(\alpha)}}{r_{2,3}^{(\alpha)}} \cdot \frac{r_{2,3}^{(\alpha)}}{r_{3,4}^{(\alpha)}} \cdots \frac{r_{N-1, N}^{(\alpha)}}{r_{N, 1}^{(\alpha)}} \cdot \frac{r_{N, 1}^{(\alpha)}}{r_{1,2}^{(\alpha)}}=1 .
\end{aligned}
$$

It is easy to see from eq. (5.5) that the constraint (5.7) holds if and only if $\exp (4 N \eta c)=1$. For the eight-vertex model, the condition has already been considered when we construct $\Phi_{c}$ in $\S 4$. Therefore, eq. (5.6) gives the only constraint on the centers $t_{(\alpha)}$ 's. From solutions of eq. (5.6) we can construct degenerate eigenvectors with the eigenvalue $(-1)^{F m_{1}} C_{0}(z)$ with the rapidities $t_{1} \ldots, t_{R}$, for any $N$ th root of unity assigned to $\exp (4 \eta c)$.

We now discuss solutions of the eq. (5.6). Let us introduce a function of variable $z$ 


$$
\begin{aligned}
G(z)= & +\sum_{a=1}^{N-1}(-1)^{r m_{1} a} e^{-4 \eta c a} \\
& \times \prod_{j=a+1}^{N}\left(\prod_{k=1}^{R} \frac{\theta\left(z-t_{k}+\eta(2 j-N+1)\right)}{\theta\left(z-t_{k}+\eta(2 j-N-3)\right)} \prod_{\beta=1}^{L} \frac{\theta\left(z-z_{\beta}+\eta(2 j-N-3)\right)}{\theta\left(z-z_{\beta}+\eta(2 j-N-1)\right)}\right)
\end{aligned}
$$

Then, the equation (5.6) of centers $t_{(\alpha)}$ 's is expressed as

$$
G\left(z=t_{(\alpha)}\right)=0, \quad \text { for } \quad \alpha=1, \ldots, F .
$$

The function $G(z)$ is an elliptic function of $z$ with periods 1 and $\tau$ when $r m_{1}$ is even, and with periods 1 and $2 \tau$ when $r m_{1}$ is odd. Making use of the Bethe ansatz equations (3.6) for $R$ rapidities $t_{1} \ldots, t_{R}$, we can show that $G(z)$ has exactly $L$ poles. It follows from the theorem of elliptic functions that $G(z)$ has $L$ zeros, which give solutions of centers $t_{(\alpha)}$ 's. For an illustration, the function $G(z)$ for the case of $N=3$ is given by

$$
G(z)=1+f(z)+\frac{1}{f(z+4 \eta)}
$$

where $f(z)$, which corresponds to $r_{1,2}^{(\alpha)} / r_{3,1}^{(\alpha)}$, is explicitly given by

$$
f(z)=(-1)^{r m_{1}} e^{4 \eta c} \prod_{k=1}^{R} \frac{\theta\left(z-t_{k}-4 \eta\right)}{\theta\left(z-t_{k}\right)} \prod_{\beta=1}^{L} \frac{\theta\left(z-z_{\beta}-2 \eta\right)}{\theta\left(z-z_{\beta}-4 \eta\right)}
$$

Let us now discuss the dimensions of degenerate eigenspaces. In fact, the $L$ zeros of $G(z)$ do not necessarily give independent eigenvectors, as we shall see shortly. For an illustration, let us consider the case of $R=0$, explicitly. Here we also assume that $L / N$ is an even integer. We can show that if $G(z)=0$, then we have $G(z+2 \eta)=0$. For example, let us consider the case of $N=3$. If $G\left(w_{1}\right)=0$, then we have $f\left(w_{1}+2 \eta\right) f\left(w_{1}+4 \eta\right) G\left(w_{1}\right)=0$. From eq. (5.10) and $f(z) f(z+2 \eta) f(z+4 \eta)=1$, we have

$$
\begin{aligned}
f\left(w_{1}+2 \eta\right) f\left(w_{1}+4 \eta\right) G\left(w_{1}\right) & =f\left(w_{1}\right) f\left(w_{1}+2 \eta\right) f\left(w_{1}+4 \eta\right)+f\left(w_{1}+2 \eta\right) f\left(w_{1}+4 \eta\right)+f\left(w_{1}+2 \eta\right) \\
& =1+\frac{1}{f\left(w_{1}\right)}+f\left(w_{1}+2 \eta\right) \\
& =G\left(w_{1}+2 \eta\right)
\end{aligned}
$$

We thus have a set of $N$ zeros of $G(z)$ as $w_{1}, w_{1}+2 \eta, \ldots, w_{1}+2(N-1) \eta$. The $L$ zeros of $G(z)$ is given by $L / N$ sets of complete $N$-strings with $L / N$ centers. Therefore, the number of independent solutions is given by $L / N$. Here we note that the $L / N$ centers should be independent in general, since the inhomogeneous parameters $z_{\beta}$ 's are generic.

Let us show that the number $2^{L / N}$ gives the dimension of the degenerate eigenspace of the XYZ model thus constructed for $R=0$ with an $N$ th root of unity assigned to $\exp (4 \eta c)$. We can calculate it by the binomial expansion

$$
2^{L / N}=\sum_{F=0}^{L / N} \frac{(L / N) !}{(L / N-F) ! F !}
$$


which corresponds to the sum of the dimensions of the degenerate subspaces with $F=$ $0,1, \ldots$, and $L / N$, respectively. Here, the dimension of the degenerate sub-eigenspace with $F$ sets of complete $N$-strings is given by the number of ways for selecting $F$ centers from the $L / N$ centers of the $L / N$ complete $N$-strings. Here we note that the factor $(-1)^{F m_{1}}$ for the eigenvalue of the transfer matrix is canceled when we take the logarithmic derivative of the eigenvalue of the transfer matrix as shown in the eq. (125) of Ref. [3].

Let us now remark that we have an extra factor $N$ for the dimension of the degenerate eigenspace of the XYZ spin chain with $R=0$, since we may choose any $N$ th root of unity for $\exp (4 \eta c)$. Thus, we obtain the number $N 2^{L / N}$ as the degeneracy in total. In fact, we can show that the degenerate eigenvalue of the XYZ Hamiltonian with $R=0$ does not depend on the choice of $c$ by using eq. (125) of Ref. [3], or more explicitly, by eq. (5.37) of Ref. [7]. For the case of $L=12$ and $N=3$, the numbers $2^{L / N}$ and $N 2^{L / N}$ are given by 16 and 48 , respectively, which are consistent with the numerical result [24].

Let us now consider the trigonometric limit for the degenerate eigenvectors of the XYZ spin chain. After taking the limit, the number of down spins $M$ becomes a good quantum number for the eigenvectors. The limit of the eigenvector $\Phi_{c}$ of the XYZ model corresponds to a linear combination of eigenvectors of the XXZ model labeled by several $M$ 's. Here we note that we replace $\lambda$ with $\lambda+\tau / 4$, and also that $\theta_{1}(z+\tau / 2) \rightarrow i \exp (-\pi z)$ and $\theta_{0}(z+\tau / 2) \rightarrow 1$ when $\tau \rightarrow i \infty$. Thus, from the expression (4.8) of $S_{L}(z, \lambda)$, we can show that the matrix elements of $S_{L}(z, \lambda)$ in the sector of $M$ down-spins have the factor $\exp (-\pi i M \lambda)$ with respect to the variable $\lambda$. Since the eigenvector $\Phi_{c}$ is given by the product of the matrix $S_{L}(z, \lambda)$ and the vector $b\left(t_{1}\right) \cdots b\left(t_{m}\right) v_{c}$, all the entries of the XXZ eigenvectors with $M$ down-spins derived from $\Phi_{c}$ have the factor $\exp ((c-\pi i(m+M)) \lambda)$ with respect to the variable $\lambda$. We now recall the sum (1.11). If $\exp (2 \eta(c-\pi i(m+M))) \neq 1$, then the XXZ eigenvectors derived from the XYZ eigenvector $\Phi_{c}$ do not have $M$ down-spins. Here we note

$$
\sum_{j=0}^{2 N-1} \exp (2 \eta j(c-\pi i(m+M)))=0 .
$$

When $\exp (2 \eta(c-\pi i(m+M)))=1$, the XXZ eigenvectors with $M$ down-spins derived from $\Phi_{c}$ do not vanish. For the degenerate eigenspace with the dimension $2^{L / N}$ for $R=0$ and $c=0$, we put $M=m$ in each sector of $m$, where $m$ is given by $m=N F$ for $F=0, \ldots, L / N$. Thus, the dimension of the degenerate XXZ eigenvectors derived from the XYZ eigenvector with $c=0$ through the trigonometric limit is given by $2^{L / N}$, in total. We note that it is equivalent to the dimension of the largest degenerate eigenspace associated with the $s l_{2}$ loop algebra of the XXZ spin chain with $L$ lattice sites in the sector $S^{Z} \equiv 0(\bmod N)$ 18], where $S^{Z}=(L-2 M) / 2$. Furthermore, we can explicitly calculate by using eq. (5.4) the trigonometric limits of the degenerate eigenvectors of the XYZ spin chain with the complete $N$-strings. Let us consider the case of $R=0$ and $c=0$ : when $F=0$ or $L / N$, the limits correspond to the degenerate XXZ eigenvectors associated with the $s l_{2}$ loop algebra [18]; when $F=1, \ldots, L / N-1$, however, we have a conjecture that the limits can be expressed as linear combinations of the degenerate XXZ eigenvectors of the complete $N$-strings, which have been shown to be associated with the $s l_{2}$ loop algebra in Refs. [19]. Thus, as a summary of the above discussion, we may conclude that at least some spectral degeneracy of the XYZ model is related to that of the XXZ model associated with the $s l_{2}$ loop algebra. 


\section{DISCUSSIONS}

We have shown that there exists a large degeneracy in the spectrum of the XYZ spin chain for the discrete $\eta$ case of $2 N \eta=m_{1}+m_{2} \tau$. The dimension $N 2^{L / N}$ of the degenerate eigenspace increases exponentially with respect to the lattice length $L$. Thus, we may have a conjecture that the spectral degeneracy of the XYZ model should have nontrivial effects on the continuum limits. We also note that in the connection between the spectra of the eight-vertex model and the six-vertex model under the trigonometric limit, the Bethe ansatz equations for the XXZ model under the twisted boundary conditions should play a central role. We shall discuss the connection explicitly in later publications.

The relation (4.2) between the $R$-matrix of $E_{\tau, \eta}\left(s l_{2}\right)$ and that of the 8VSOS model is fundamental in the elliptic quantum groups. We may interpret that the $R$-matrix of $E_{\tau, \eta}\left(s l_{2}\right)$ is derived from that of the $8 \mathrm{VSOS}$ model through the relation. In fact, we can discuss the $R$ matrices of the elliptic quantum groups associated with the ABCD-type Lie algebras [12] based on the Boltzmann weights [25,26] of the ABCD IRF models.

As an application of the observation in the last paragraph, we can discuss the spectral degeneracy of the $A$-type IRF models [25] through the nested algebraic Bethe ansatz of the associated elliptic quantum groups. Here we note that the $A$-type IRF models are related to the elliptic vertex model [27], which corresponds to the higher-rank extension of the eightvertex model, through the vertex-IRF correspondence. This approach should be related to the higher-rank loop-algebra symmetry of the vertex models discussed in Ref. [28].

Finally, we remark that it should be straightforward to discuss the spectral degeneracy for the higher-spin generalization of the XYZ model by making use of the higher-spin version [29] of the vertex-IRF correspondence.

\section{Acknowledgements}

The author would like to thank Prof. K. Fabricius and Prof. B.M. McCoy for helpful discussions and valuable comments. He would also like to thank Prof. M. Kashiwara

and Prof. T. Miwa for their kind invitation to the conference "MathPhys Odyssey 2001", Okayama-Kyoto, February, 2001, where quite an early version of this paper has been presented. He is thankful to many participants of the conference for their helpful comments. This work is partially supported by the Grant-in-Aid for Encouragement of Young Scientists (No. 12740231). 


\section{A. APPENDIX: THE $S L_{2}$ LOOP ALGEBRA}

Let us introduce the loop algebra for $\operatorname{sl}(2, \mathbf{C})$. The loop algebra associated to $s l_{2}$ consists of the space of analytic mappings from the circle $S^{1}$ to $s l_{2}$. If $T^{a}$ is a basis of $s l_{2}$, and $S^{1}$ is considered as the unit circle in the complex plane with coordinate $z=\exp (2 \pi i t)$, then Fourier analysis shows that a topological basis of the vector space of these maps is given by $T^{a} \otimes z^{n}$ for $n \in \mathbf{Z}[30]$. The commutation relation is defined by $\left[T^{a} \otimes z^{m}, T^{b} \otimes z^{n}\right]=\left[T^{a}, T^{b}\right] \otimes z^{m+n}$. The affine Lie algebra $\hat{s l_{2}}$ is given by the central extension of the $s l_{2}$ loop algebra together with the derivation $d: s l_{2}=s l_{2} \otimes \mathbf{C}\left[z, z^{-1}\right] \oplus \mathbf{C} c \oplus \mathbf{C} d$, where the commutation relations are given by

$$
\begin{aligned}
{\left[T^{a} \otimes z^{m}, T^{b} \otimes z^{n}\right] } & =\left[T^{a}, T^{b}\right] \otimes z^{m+n}+m \delta_{m+n, 0} \operatorname{tr}\left(T^{a} T^{b}\right) c, \\
{\left[d, T^{a} \otimes z^{m}\right] } & =m T^{a} \otimes z^{m}, \quad\left[c, T^{a} \otimes z^{m}\right]=0 .
\end{aligned}
$$

We shall also consider the affine algebra with no derivation $d: \hat{s l}_{2}^{\prime}=s l_{2} \otimes \mathbf{C}\left[z, z^{-1}\right] \oplus \mathbf{C} c$.

Let us denote the Chevalley generators of the $s l_{2}$ by $e, f$ and $h$. Then, we may define the Chevalley generators of $\hat{s l}_{2}$ by the following: $h_{0}=c-h \otimes 1, h_{1}=h \otimes 1, e_{0}=f \otimes z$, $f_{0}=e \otimes z^{-1}, e_{1}=e \otimes 1, f_{1}=f \otimes 1$ and $d=t \partial / \partial t$. The Cartan subalgebra $\hat{\mathbf{h}}$ of $\hat{s l_{2}}$ is spanned by $h_{0}, h_{1}$ and $d$. In terms of the Chevalley basis, the defining relations of $\hat{s l}_{2}$ are given by

$$
\begin{aligned}
{\left[h_{0}, h_{1}\right] } & =0, \quad\left[d, h_{j}\right]=0 \quad(j=0,1) \\
{\left[h_{i}, e_{j}\right] } & =a_{i j} e_{j}, \quad\left[h_{i}, f_{j}\right]=-a_{i j} f_{j}, \quad\left[e_{i}, f_{j}\right]=\delta_{i j} h_{j}, \quad(i, j=0,1) \\
{\left[d, e_{j}\right] } & =\delta_{0, j} e_{j}, \quad\left[d, f_{j}\right]=-\delta_{0 j} f_{j}, \quad(j=0,1) \\
{\left[e_{i},\left[e_{i},\left[e_{i}, e_{j}\right]\right]\right] } & =0, \quad\left[f_{i},\left[f_{i},\left[f_{i}, f_{j}\right]\right]=0 \quad(i, j=0,1, \quad i \neq j)\right.
\end{aligned}
$$

Here, the Cartan matrix $\left(a_{i j}\right)$ of $A_{1}^{(1)}$ is defined by

$$
\left(\begin{array}{ll}
a_{00} & a_{01} \\
a_{10} & a_{11}
\end{array}\right)=\left(\begin{array}{cc}
2 & -2 \\
-2 & 2
\end{array}\right)
$$

Let us now review the connection of the $s l_{2}$ loop algebra to the XXZ Hamiltonian $H_{X X Z}$ at the root of unity. Hereafter, we assume that $q^{2 N}=1$. We introduce the operators $S^{ \pm(N)}$ by

$$
\begin{aligned}
S^{ \pm(N)}= & \sum_{1 \leq j_{1}<\cdots<j_{N} \leq L} q^{\frac{N}{2} \sigma^{Z}} \otimes \cdots \otimes q^{\frac{N}{2} \sigma^{Z}} \otimes \sigma_{j_{1}}^{ \pm} \otimes q^{\frac{(N-2)}{2} \sigma^{Z}} \otimes \cdots \otimes q^{\frac{(N-2)}{2} \sigma^{Z}} \\
& \otimes \sigma_{j_{2}}^{ \pm} \otimes q^{\frac{(N-4)}{2} \sigma^{Z}} \otimes \cdots \otimes \sigma_{j_{N}}^{ \pm} \otimes q^{-\frac{N}{2} \sigma^{Z}} \otimes \cdots \otimes q^{-\frac{N}{2} \sigma^{Z}} .
\end{aligned}
$$

We denote by $T^{ \pm(N)}$ the operators obtained from $S^{ \pm(N)}$ via replacing $q$ with $q^{-1}$. Explicitly, we have

$$
\begin{aligned}
T^{ \pm(N)}= & \sum_{1 \leq j_{1}<\cdots<j_{N} \leq L} q^{-\frac{N}{2} \sigma^{Z}} \otimes \cdots \otimes q^{-\frac{N}{2} \sigma^{Z}} \otimes \sigma_{j_{1}}^{ \pm} \otimes q^{-\frac{(N-2)}{2} \sigma^{Z}} \otimes \cdots \otimes q^{-\frac{(N-2)}{2} \sigma^{Z}} \\
& \otimes \sigma_{j_{2}}^{ \pm} \otimes q^{-\frac{(N-4)}{2} \sigma^{Z}} \otimes \cdots \otimes \sigma_{j_{N}}^{ \pm} \otimes q^{\frac{N}{2} \sigma^{Z}} \otimes \cdots \otimes q^{\frac{N}{2} \sigma^{Z}}
\end{aligned}
$$


Let the symbol $T_{6 V}(v)$ denotes the (inhomogeneous) transfer matrix of the six-vertex model. Here we recall that $S^{Z}$ denotes the $Z$-component of the total spin operator. Then we can show the (anti) commutation relations in the sector of $S^{Z} \equiv 0(\bmod \mathrm{N})$ [18]

$$
S^{ \pm(N)} T_{6 V}(v)=q^{N} T_{6 V}(v) S^{ \pm(N)}, \quad T^{ \pm(N)} T_{6 V}(v)=q^{N} T_{6 V}(v) T^{ \pm(N)}
$$

Since the XXZ Hamiltonian $H_{X X Z}$ is given by the logarithmic derivative of the (homogeneous) transfer matrix $T_{6 V}(v)$, we have in the sector $S^{Z} \equiv 0(\bmod N)$

$$
\left[S^{ \pm(N)}, H_{X X Z}\right]=\left[T^{ \pm(N)}, H_{X X Z}\right]=0 .
$$

Thus, the operators $S^{ \pm(N)}$ and $T^{ \pm(N)}$ commute with the XXZ Hamiltonian in the sector $S^{Z} \equiv 0(\bmod N)$.

Let us now consider the algebra generated by the operators $S^{ \pm(N)}$ and $T^{ \pm(N)}$ [18. When $q$ is a primitive $2 N$ th root of unity, or a primitive $N$ th root of unity with $N$ odd, we can show that $S^{ \pm(N)}$ and $T^{ \pm(N)}$ satisfy the relations in the following [18]:

$$
\begin{gathered}
{\left[S^{+(N)}, T^{+(N)}\right]=\left[S^{-(N)}, T^{-(N)}\right]=0,} \\
{\left[S^{Z}, S^{ \pm(N)}\right]= \pm N S^{ \pm(N)}, \quad\left[S^{Z}, T^{ \pm(N)}\right]= \pm N T^{ \pm(N)},} \\
\left(S^{+(N)}\right)^{3} T^{-(N)}-3\left(S^{+(N)}\right)^{2} T^{-(N)} S^{+(N)}+3 S^{+(N)} T^{-(N)}\left(S^{+(N)}\right)^{2}-T^{-(N)}\left(S^{+(N)}\right)^{3}=0, \\
\left(S^{-(N)}\right)^{3} T^{+(N)}-3\left(S^{-(N)}\right)^{2} T^{+(N)} S^{-(N)}+3 S^{-(N)} T^{+(N)}\left(S^{-(N)}\right)^{2}-T^{+(N)}\left(S^{-(N)}\right)^{3}=0, \\
\left(T^{+(N)}\right)^{3} S^{-(N)}-3\left(T^{+(N)}\right)^{2} S^{-(N)} T^{+(N)}+3 T^{+(N)} S^{-(N)}\left(T^{+(N)}\right)^{2}-S^{-(N)}\left(T^{+(N)}\right)^{3}=0, \\
\left(T^{-(N)}\right)^{3} S^{+(N)}-3\left(T^{-(N)}\right)^{2} S^{+(N)} T^{-(N)}+3 T^{-(N)} S^{+(N)}\left(T^{-(N)}\right)^{2}-S^{+(N)}\left(T^{-(N)}\right)^{3}=0,
\end{gathered}
$$

and in the sector $S^{z} \equiv 0(\bmod N)$ we have

$$
\left[S^{+(N)}, S^{-(N)}\right]=\left[T^{+(N)}, T^{-(N)}\right]=-(-q)^{N} \frac{2}{N} S^{z} .
$$

When $q$ is a primitive $2 N$ th root of unity with $N$ even, or when $q$ is a primitive $N$ th root of unity with $N$ odd, then, we consider the identification in the following:

$$
e_{0}=S^{+(N)}, \quad f_{0}=S^{-(N)}, \quad e_{1}=T^{-(N)}, \quad f_{1}=T^{+(N)}, \quad h_{0}=-h_{1}=\frac{2}{N} S^{z} .
$$

We see that the operators $e_{j}, f_{j}, h_{j}$ for $j=0,1$, satisfy the defining relations (A.2) of the algebra $\hat{s l}_{2}^{\prime}$ with $c=0$. The relations (A.8) and (A.11) correspond to some relations of (A.2) in the following: $\left[h_{i}, e_{j}\right]=a_{i j} e_{j},\left[h_{i}, f_{j}\right]=-a_{i j} f_{j}$ and $\left[e_{i}, f_{j}\right]=\delta_{i j} h_{j}$. The relations (A.10) correspond to the Serre relations of (A.2). Thus, they give a representation of the $s l_{2}$ loop algebra or a finite-dimensional representation of $\hat{s l}_{2}^{\prime}$.

When $q$ is a primitive $2 N$ th root of unity with $N$ odd, we may put as follows

$$
e_{0}=i S^{+(N)}, \quad f_{0}=i S^{-(N)}, \quad e_{1}=i T^{-(N)}, \quad f_{1}=i T^{+(N)}, \quad h_{0}=-h_{1}=\frac{2}{N} S^{z} .
$$

We see that they give a representation of the $s l_{2}$ loop algebra or a finite-dimensional representation of $\hat{s l}_{2}^{\prime}$.

Finally we note that the loop algebras with higher ranks are discussed for some vertex models [28]. 


\section{B. APPENDIX: EVALUATION MODULES OF THE ELLIPTIC QUANTUM GROUP}

We first define a diagonalizable $\mathbf{h}$-module [13, 14]. Let $V$ be a module over the onedimensional Lie algebra $\mathbf{h}$ with the generator $h$ such that $V$ is the direct sum of finite dimensional eigenspaces $V[\mu]$ of $h$, labeled by the eigenvalue $\mu$. We call such a module a diagonalizable $\mathbf{h}$-module.

Let us now define a module over the elliptic quantum group $E_{\tau, \eta}\left(s l_{2}\right)$ [13, 14. An $E_{\tau, \eta}\left(s l_{2}\right)$ module is a diagonalizable $\mathbf{h}$-module $V$ together with a meromorphic function $L(z, \lambda)$ on $\mathbf{C} \otimes \mathbf{h}$ with values in $\operatorname{End}\left(\mathbf{C}^{2} \otimes V\right)$ such that the dynamical Yang-Baxter relation (2.1) holds in $\operatorname{End}\left(\mathbf{C}^{2} \otimes \mathbf{C}^{2} \otimes V\right)$.

When $V=\mathbf{C}^{2}, L(z, \lambda)=R\left(z-z_{0}, \lambda\right)$ gives a module over $E_{\tau, \eta}\left(s l_{2}\right)$, which we call the fundamental representation with evaluation point $z_{0}$. More generally, for any pair of complex numbers, $\Lambda$, $z$, we can define an evaluation Verma module $W_{\Lambda}(z)$ as follows [13]. Let $W_{\Lambda}(z)$ be an infinite dimensional complex vector space with a basis $e_{k}\left(k \in \mathbf{Z}_{\geq 0}\right)$. We define an action of $f(h)$ by

$$
f(h) e_{k}=f(\Lambda-2 k) e_{k} \quad(k=0,1, \ldots)
$$

and that of the other generators by

$$
\begin{aligned}
& a(w, \lambda) e_{k}=\frac{\theta(z-w+(\Lambda+1-2 k) \eta)}{\theta(z-w+(\Lambda+1) \eta)} \frac{\theta(\lambda+2 k \eta)}{\theta(\lambda)} e_{k} \\
& b(w, \lambda) e_{k}=-\frac{\theta(-\lambda+z-w+(\Lambda-1-2 k) \eta)}{\theta(z-w+(\Lambda+1) \eta)} \frac{\theta(2 \eta)}{\theta(\lambda)} e_{k+1}, \\
& c(w, \lambda) e_{k}=-\frac{\theta(-\lambda-z+w+(\Lambda+1-2 k) \eta)}{\theta(z-w+(\Lambda+1) \eta)} \frac{\theta(2(\Lambda+1-k) \eta)}{\theta(\lambda)} \frac{\theta(2 k \eta)}{\theta(2 \eta)} e_{k-1}, \\
& d(w, \lambda) e_{k}=\frac{\theta(z-w+(-\Lambda+1+2 k) \eta)}{\theta(z-w+(\Lambda+1) \eta)} \frac{\theta(\lambda-2(\Lambda-k) \eta)}{\theta(\lambda)} e_{k} .
\end{aligned}
$$

It is shown in Ref. [13 that these formulas define an $E_{\tau, \eta}\left(s l_{2}\right)$-module structure on $W_{\Lambda}(z)$; if $\Lambda=n+(m+\ell \tau) / 2 \eta$, where $n, m$ and $\ell$ are integers with $n \geq 0$, then the space spanned by $e_{k}, k>n$, is a submodule. The quotient space $L_{\Lambda}(z)$ is a module of dimension $n+1$.

The evaluation Verma module $W_{\Lambda}(z)$ is a highest weight module with highest weight vector $e_{0}$ and highest weight $(\Lambda, A(w, \lambda), D(w, \lambda))$, where $A(w, \lambda)=1$, and

$$
D(w, \lambda)=\frac{\theta(z-w+(-\Lambda+1) \eta)}{\theta(z-w+(\Lambda+1) \eta)} \frac{\theta(\lambda-2 \Lambda \eta)}{\theta(\lambda)}
$$

Let us denote the finite dimensional module $L_{\Lambda}(z)$ by $V_{\Lambda}(z)$ if $\Lambda=n+(m+\ell \tau) / 2 \eta$ where $n, m, \ell$ are integers with $n \geq 0$, and the infinite dimensional module $W_{\lambda}(z)$ if $\Lambda$ does not have this form. Then, the following proposition plays an important role in $\S 3$.

Proposition B.1 [13] Let $W=V_{\Lambda_{1}}\left(z_{1}\right) \otimes \cdots V_{\Lambda_{n}}\left(z_{n}\right)$ be the tensor product of evaluation modules $W_{\Lambda_{j}}\left(z_{j}\right)$ 's, and let $\Lambda=\Lambda_{1}+\Lambda_{2}+\cdots+\Lambda_{n}$. Then, $W[\Lambda]=\mathbf{C} v_{0}$, and for every $z$ we have 


$$
a(z) v_{0}=A(z, \lambda) v_{0}, \quad c(z) v_{0}=0, \quad d(z) v_{0}=D(z, \lambda) v_{0}
$$

where

$$
A(z, \lambda)=1, \quad D(z, \lambda)=\frac{\theta(\lambda-2 \Lambda)}{\theta(\lambda)} \prod_{j=1}^{n} \frac{\theta\left(z-p_{j}\right)}{\theta\left(z-q_{j}\right)}
$$

Here $p_{j}=z_{j}+\eta\left(-\Lambda_{j}+1\right)$ and $q_{j}=z_{j}+\eta\left(\Lambda_{j}+1\right)$.

\section{APPENDIX: THE IRF MODELS RELATED TO THE EIGHT-VERTEX MODEL}

Let us consider a two-dimensional square lattice with a spin variable $a_{i}$ associated to each site $i$ [21]. We shall call the $a_{i}$ a state and assume that $a_{i} \in S$ with $S$ being a set of the states. The set $S$ is finite for restricted Solid-on-Solid models (RSOS models), while it is infinite for unrestricted Solid-on-Solid models (unrestricted SOS models). Here we note that unrestricted and restricted SOS models are also called Interaction-Round-a-Face models or IRF models, briefly [5].

Let us consider the case of RSOS models. Let $s$ denote the number of elements in $S$. Consider a $s \times s$ matrix $C$ satisfying the following conditions 21,22]:

(i) $C_{a b}=C_{b a}=0$ or 1

(ii) $C_{a a}=0$

(iii) For each $a \in S$, there should exist $b \in S$ such that $C_{a b}=1$

For such choice of $C$, we impose a restriction that two states $a$ and $b$ can occupy the neighboring lattice sites if and only if $C_{a b}=1$. We call such a pair of the states $(a, b)$ admissible. For the case of unrestricted models, the infinite matrix $C$ satisfies the conditions (i), (ii), and (iii) with an infinite set $S$.

For an illustration, let us consider the restricted eight-vertex Solid-on-Solid model (the restricted 8V SOS model), which we also call the ABF model [15]. For the $N$-state case, we have $S=\{1,2, \ldots, N\}$. The nonzero matrix elements of $C$ are given by $C_{j, j+1}=C_{j+1, j}=1$ for $j=1,2, \ldots, N-1$; other matrix elements such as $C_{1, N}$ and $C_{N, 1}$ are given by zero.

Let $a_{i}, a_{j}, a_{k}$, and $a_{\ell}$ be the four states assigned on the lattice sites $i, j, k$, and $\ell$ surrounding a face. Here $i, j, k, \ell$ are ordered counterclockwise from the southwest corner. We assume that an elementary configuration is given by the configuration of the four spin variables around the face and the probability of having $a_{i}, a_{j}, a_{k}, a_{\ell}$ around the face is denoted by the Boltzmann weight $w\left(a_{i}, a_{j}, a_{k}, a_{\ell} ; z\right)$. Here the variable $z$ is called the spectral parameter. The model is called solvable if the Boltzmann weights satisfy the Yang-Baxter relations in the following:

$$
\begin{aligned}
& \sum_{g} w(a, b, g, f ; z-w) w(f, g, d, e ; z) w(g, b, c, d ; w) \\
= & \sum_{g} w(f, a, g, e ; w) w(a, b, c, g ; z) w(g, c, d, e ; z-w),
\end{aligned}
$$

where the summation of the variable $g$ is taken over all the admissible states.

Let us introduce explicitly some solutions of the Yang-Baxter relations. We consider a slightly generalized version of eq. (4.2), where $\lambda$ is given by $\lambda=-2 \eta d-w_{0}$ with some 
parameter $w_{0}$. Then, we have the Boltzmann weights $w\left(a, b, c, d ; z, w_{0}\right)$ of the unrestricted eight-vertex Solid-on-Solid model (unrestricted 8V SOS model)

$$
\begin{aligned}
w\left(d+1, d+2, d+1, d ; z, w_{0}\right) & =w(d, d-1, d-2, d-1 ; z)=1 \\
w\left(d-1, d, d+1, d ; z, w_{0}\right) & =\alpha\left(z,-2 \eta d-w_{0}\right) \\
w\left(d+1, d, d-1, d ; z, w_{0}\right) & =\alpha\left(z, 2 \eta d+w_{0}\right) \\
w\left(d+1, d, d+1, d ; z, w_{0}\right) & =\beta\left(z, 2 \eta d+w_{0}\right) \\
w\left(d-1, d, d-1, d ; z, w_{0}\right) & =\beta\left(z,-2 \eta d-w_{0}\right)
\end{aligned}
$$

The Boltzmann weights (C.2) satisfy the Yang-Baxter relations (C.1).

Let us introduce a gauge transformation

$$
w(a, b, c, d ; z) \rightarrow w(a, b, c, d ; z) \frac{g_{c}}{g_{a}} .
$$

Then, we see that the transformed Boltzmann weights also satisfy the Yang-Baxter relations (C.1). Setting $g_{a}=\exp (\pi i a / 2) \sqrt{\theta\left(2 \eta a+w_{0}\right)}(a \in \mathbf{Z})$, applying (C.3) to (C.2), and multiplying the Boltzmann weights by $\rho(z)=\theta(2 \eta-z) / \theta(2 \eta)$, we have the standard expressions of the Boltzmann weights such as in Refs. [15,20 22] in the following:

$$
\begin{aligned}
w\left(d+1, d+2, d+1, d ; z, w_{0}\right) & =w(d, d-1, d-2, d-1 ; z)=\frac{\theta(2 \eta-z)}{\theta(2 \eta)} \\
w\left(d-1, d, d+1, d ; z, w_{0}\right) & =w\left(d+1, d, d-1, d ; z, w_{0}\right) \\
& =\frac{\theta(z)}{\theta(2 \eta)} \frac{\sqrt{\theta\left(2 \eta(d+1)+w_{0}\right) \theta\left(2 \eta(d-1)+w_{0}\right)}}{\theta\left(2 \eta d+w_{0}\right)} \\
w\left(d+1, d, d+1, d ; z, w_{0}\right) & =\frac{\theta\left(z+2 \eta d+w_{0}\right)}{\theta\left(2 \eta d+w_{0}\right)} \\
w\left(d-1, d, d-1, d ; z, w_{0}\right) & =\frac{\theta\left(z-2 \eta d-w_{0}\right)}{\theta\left(2 \eta d+w_{0}\right)}
\end{aligned}
$$

Let us now consider the Boltzmann weights of the ABF and CSOS models. Here we assume that $2 N \eta=m_{1}$, where integer $m_{1}$ has no common divisor with $N$. If we set $w_{0}=0$, then we have the Boltzmann weights of the ABF model. We can show that the Boltzmann weights (C.4) satisfy the Yang-Baxter relations (C.1) with the finite set: $S=\{1,2, \ldots, N\}$. If we set $w_{0} \neq 0$, then we have the Boltzmann weights of the cyclic SOS model (CSOS model). We can show that the Boltzmann weights (C.4) satisfy the Yang-Baxter relations with the finite set: $S=\{1,2, \ldots, N\}$ and the cyclic admissible conditions: $C_{1, N}=C_{N, 1}=1$. Here we note this RSOS model is called the cyclic SOS model in Ref. [20], the $A_{N-1}^{(1)}$ model in Ref. [21], and the periodic 8V SOS model in Ref. [22] (See also the Appendix of Ref. [22]). 


\section{REFERENCES}

[1] R. Baxter, Ann. Phys. 70 (1972) 193.

[2] R. Baxter, Ann. Phys. 76 (1973) 1.

[3] R. Baxter, Ann. Phys. 76 (1973) 25.

[4] R. Baxter, Ann. Phys. 76 (1973) 48.

[5] R.J. Baxter, Exactly Solved Models in Statistical Mechanics (Academic Press, London, 1982).

[6] Yang-Baxter equation in integrable systems ed. by M. Jimbo (World scientific, Singapore, 1990).

[7] L. Takhtajan and L. Faddeev, Russ. Math. Survey 34(5) (1979) 11.

[8] J.D. Johnson, S. Krinsky and B.M. McCoy, Phys. Rev. A 8 (1973) 2526.

[9] A. Luther, Phys. Rev. B (1976) 14 (1976) 2153

[10] S. Pokrovsky and A.M. Tsvelik, Sov. JETP 93 (1987) 2232.

[11] I. Affleck, Fields, Strings and Critical Phenomena ed. E. Brezin and J. Zinn-Justin (1990, Amsterdam, North-Holland) 563.

[12] G. Felder, in the Proceedings of the International Congress of Mathematicians, Zürich, 1994 (Birkhäuser, Basel, 1994) p.1247.

[13] G. Felder and A. Varchenko, Commun. Math. Phys. 181(1996) 741 .

[14] G. Felder and A. Varchenko, Nucl. Phys. B 480 (1996) 485.

[15] G.E. Andrews, R.J. Baxter and P.J. Forrester, J. Stat. Phys. 35 (1984) 193.

[16] O. Babelon, D. Bernard, E. Billey, Phys. Lett. B 375 (1996) 89.

[17] M. Jimbo, H. Konno, S. Odake, J. Shiraishi, Transformation Groups 4, 303 (1999).

[18] T. Deguchi, K. Fabricius and B.M. McCoy, J. Stat. Phys. 102 (2001) 701.

[19] K. Fabricius and B.M. McCoy, J. Stat. Phys. 103(2001) 647; J. Stat. Phys. 104(2001) 575; cond-mat/0108057.

[20] P.A. Pearce and K.A. Seaton, Phys. Rev. Lett. 60 (1988) 1347.

[21] A. Kuniba and T. Yajima, J. Stat. Phys. 52, 829 (1987).

[22] Y. Akutsu, T. Deguchi and M. Wadati, J. Phys. Soc. Jpn. 57, 1173 (1988).

[23] T. Deguchi, J. Phys. A: Math. Gen. 34 (2001) 9755.

[24] K. Fabricius, a private communication.

[25] M. Jimbo, T. Miwa and M. Okado, Nucl. Phys. B 300 (1988) 74.

[26] M. Jimbo, T. Miwa and M. Okado, Commun. Math. Phys. 116 (1988) 353.

[27] A.A. Belavin, Nucl. Phys. B 180 (1981) 189.

[28] C. Korff and B.M. McCoy, hep-th/0104120.

[29] T. Takebe, J. Phys. A: Math. Gen. 28(1995) 6675; J. Phys. A: Math. Gen. 29 (1996) 1563.

[30] J. Fuchs and C. Schweigert, Symmetries, Lie Algebras and Representations, (Cambridge Univ. Press, 1997). 